\title{
Intravitreal Aflibercept injection with Panretinal photocoagulation versus early Vitrectomy for diabetic vitreous hemorrhage: randomized clinical trial
}

\author{
Ahmed Hosni Abd Elhamid ${ }^{1,2 *}$, Ahmed Abd El Alim Mohamed ${ }^{1}$ and Abeer Mohamed Khattab 2,3
}

\begin{abstract}
Background: To compare efficacy and safety of intravitreal aflibercept (IVA) injection with panretinal photocoagulation (PRP) versus early vitrectomy for diabetic vitreous hemorrhage (VH).

Methods: Prospective, randomized study that included 34 eyes with diabetic VH. They were divided into two groups, Group I (17 eyes) received three successive IVA injections followed by PRP and group II (17 eyes) for whom early vitrectomy was done. Follow up was carried out after one, two, three, six and nine months. The primary outcome measure was change in the mean best corrected visual acuity (BCVA) after nine months, secondary outcome measures were mean duration of clearance of $\mathrm{VH}$ and rate of recurrent hemorrhage with any additional treatment in both groups. Complications were reported.
\end{abstract}

Results: There was no statistically significant difference regarding initial demographic criteria between both groups. The mean final log MAR BCVA was statistically better than the initial BCVA in both groups $(0.51 \pm 0.20,1.17 \pm 0.48$ for group I and $0.48 \pm 0.18,1.44 \pm 0.44$ for group $I I, P<0.001)$. There was no statistically significant difference between both groups regarding the mean final Log Mar BCVA $(0.51 \pm 0.20$ for group I, $0.48 \pm 0.18$ for group II, $p \geq$ 0.05), the mean duration of clearance of $\mathrm{VH}$ was $7.8 \pm 1.8$ weeks, 5 days for group I and II respectively. PRP was completely done for all eyes in group I after three months. The difference in the recurrence rate between group I (29.4\%) and group II (11.8\%) was statistically significant $(p<0.05)$. Vitrectomy was done for three eyes $(17.6 \%)$ due to recurrent non-resolving $\mathrm{VH}$ in group I. late recurrent VH occurred in two eyes (11.8\%) in group II, IVA was given with complete clearance of the hemorrhage. No vision threatening complications were reported in both groups.

Conclusion: Both intravitreal injection of aflibercept followed by PRP and early vitrectomy are effective and safe modalities for treatment of diabetic vitreous hemorrhage. Early vitrectomy leads to faster vision gain with less incidence of recurrence than intravitreal injection.

Trial registration: Randomized clinical trial under the number of NCT04153253 on November 6, 2019 "Retrospectively registered".

Keywords: Vitreous hemorrhage, Intravitreal injection, Panretinal photocoagulation, Pars plana vitrectomy

\footnotetext{
* Correspondence: hosni712000@yahoo.com

'Ophthalmology Department, Ain Shams University, Cairo, Egypt

${ }^{2}$ Affiliated as vitreoretinal consultant, Hadi hospital, Jabriya, Kuwait

Full list of author information is available at the end of the article
}

(C) The Author(s). 2020 Open Access This article is licensed under a Creative Commons Attribution 4.0 International License, which permits use, sharing, adaptation, distribution and reproduction in any medium or format, as long as you give appropriate credit to the original author(s) and the source, provide a link to the Creative Commons licence, and indicate if changes were made. The images or other third party material in this article are included in the article's Creative Commons licence, unless indicated otherwise in a credit line to the material. If material is not included in the article's Creative Commons licence and your intended use is not permitted by statutory regulation or exceeds the permitted use, you will need to obtain permission directly from the copyright holder. To view a copy of this licence, visit http://creativecommons.org/licenses/by/4.0/ The Creative Commons Public Domain Dedication waiver (http://creativecommons.org/publicdomain/zero/1.0/) applies to the data made available in this article, unless otherwise stated in a credit line to the data. 


\section{Background}

Diabetic retinopathy is the leading cause of vision loss in working-aged adults worldwide. In proliferative diabetic retinopathy (PDR) stage, hypoxic retinal tissue leads to liberation of angiogenic agents such as vascular endothelial growth factor (VEGF) which plays a major role in the development of retinal and optic disc neovascularization, such neo vessels can bleed at any time and cause vitreous hemorrhage $(\mathrm{VH})$. Although $\mathrm{VH}$ occurring as a complication of PDR can cause acute and sometimes severe vision deterioration, there is no current, evidencebased clinical guidance as to what is the best treatment method to provide the best visual outcomes once intervention is needed [1].

For years, pan retinal photocoagulation (PRP) has been the standard of care for high risk PDR and has been shown to reduce severe vision loss by more than $50 \%$. If diabetic $\mathrm{VH}$ occurs before detection of the neovascularization, it may be very difficult to do PRP as retinal details are not visible [2].

Anti-VEGF therapy has been shown to cause regression of neovascularization and to potentially prevent further hemorrhage [3]. Recent study from the diabetic retinopathy clinical research network (DRCRN) reported that intravitreal ranibizumab (IVR) is non-inferior to PRP treatment for PDR at the end of two years but the study did not address the role in $\mathrm{VH}$ [4]. Another multicenter study compared the outcome of IVR injections versus intravitreal saline injections in the treatment of $\mathrm{VH}$ in patients with PDR, and suggested that IVR increased the likelihood of completion of PRP without pars plana vitrectomy (PPV) [5]. Also, there was a beneficial effect of intravitreal bevacizumab (IVB) for managing $\mathrm{VH}$ in PDR [6].

PPV is indicated if the hemorrhage does not clear on its own, but surgery does not preclude the possibility of recurrent $\mathrm{VH}$, which has been reported to occur in 20 $40 \%$ of eyes [7]. Diabetic retinopathy vitrectomy study (DRVS) concluded that there is a benefit of early vitrectomy in eyes of type I diabetics with severe $\mathrm{VH}$ and the majority (80\%) of patients with type II diabetes with severe $\mathrm{VH}$ still require vitrectomy to resolve the $\mathrm{VH}$ after 1 year [8]. DRVS was conducted before the development of endolaser photocoagulation which assures complete coagulation of the retina. Further, wide-field visualization, which evolved in the late 1990s, enables more complete endolaser photocoagulation treatment and may allow for better outcomes [9]. Recent studies showed better visual outcome for vitrectomy than results of DRVS, One study showed that the mean visual acuity improved from $20 / 600$ preoperatively to $20 / 90$ postoperatively [10] .

The development of sutureless, micro-incision $(23,25$ and 27 gauge) PPV has resulted in less inflammation, increased patient comfort and faster recovery in the post-operative period. Also, with less operative time, the type of anesthesia has been changed from general into local in most of the cases. The improved visual and anatomic outcomes of PPV with current techniques and the reduced post-operative discomfort can encourage retinal surgeons to perform PPV for diabetic $\mathrm{VH}$ earlier than three months, which had once been the widely adopted standard for observation $[11,12]$.

The aim of the study is to compare the efficacy and safety of two different treatment options for diabetic $\mathrm{VH}$, intravitreal aflibecept injection (IVA) with PRP versus early PPV.

\section{Methods}

Prospective randomized study that included patients presenting with diabetic $\mathrm{VH}$ in the period from March 2018 to July 2019. The study was done in two eye centers; Hadi hospital in Kuwait and Ain Shams University hospital in Egypt. The study was performed in accordance with the ethical standards of the Declaration of Helsinki and was approved by the Ethical Committee of Hadi hospital.

\section{Study population}

Thirty four eyes of 34 patients with $\mathrm{VH}$ as a complication of PDR were selected. Eligibility criteria were age above 18 years, any sex, type I or II diabetes mellitus (DM), recent diabetic $\mathrm{VH}$ causing vision impairment and precluding complete PRP, and best corrected visual acuity (BCVA) less than 20/80 (equivalent to log MAR BCVA score of 0.6) and better than 20/1000 (log MAR BCVA of 1.7). Exclusion criteria were tractional retinal detachment, previous PRP, history of anti-VEGF therapy within the past two months, neo vascular glaucoma (patients with iris neo vessels and normal intraocular pressure (IOP) were not excluded), subhyaloid clotted hemorrhage, vitreomacular traction and raised fibro vascular proliferations. Patients with diabetic macular edema (DME) at time of presentation (if it can be diagnosed) and those diagnosed at any time of the study whether after IVA injection or after surgery were excluded. Patients with systemic contraindications for anti-VEGF or unstable medical conditions such as uncontrolled hypertension (persistently above 180/110 $\mathrm{mmHg}$ ) or recent thromboembolic event within the past six months were also excluded from the study.

\section{Initial assessment}

BCVA was measured using Snellen chart which was converted into log MAR, anterior segment slit lamp examination, IOP measurement by Goldmann applanation tonometry, detailed fundus examination with indirect ophthalmoscopy and bio microscopy using $90 \mathrm{D}$ lens if 
possible. B scan ultrasound is required to exclude tractional retinal detachment if details of the retina were not clear by clinical examination.

\section{Grading of VH \\ Grade I}

Mild VH included patients with hazy media, clear or a blurry nerve fiber layer and clear optic disc and retinal vessels.

\section{Grade II}

Moderate VH included all patients with non-visible nerve fiber layer, blurry optic disc and blurry retinal vessels.

\section{Grade III}

Severe VH included patients with neither visible optic disc nor retinal vessels.

\section{Patients' allocation}

Eyes were randomly allocated into either groups by simple coin flip. Group I (17 eyes) was subjected to three monthly doses of IVA injections (EYLEA ${ }^{\circ}$; Regeneron Pharmaceutical Inc., Tarrytown, NY, USA and Bayer Healthcare, Berlin, Germany) in a dose of $2 \mathrm{mg} / 0.05 \mathrm{ml}$. The first injection was given within a week after presentation. PRP was done after the third injection in case of VH clearance. Group II (17 eyes) was subjected to PPV with endolaser treatment within one week of presentation.

\section{Interventional techniques \\ Intravitreal injection (IVI)}

The technique was done in a sterile operating room. After sterilization and toweling with application of sterile speculum, topical anesthetic (oxybuprocain 0.4\%) eye drops was instilled into the eye to be treated. Povidone iodine $5 \%$ was instilled into the conjunctiva. Injection of $2 \mathrm{mg} / 0.05 \mathrm{ml}$ aflibercept was given $4 \mathrm{~mm}$ from the limbus in phakic eyes and $3.5 \mathrm{~mm}$ in pseudophakic eyes. Vision was checked at the end of the procedure to be at least light perception. Topical antibiotic eye drops were given for five days.

\section{Panretinal photocoagulation (PRP)}

The treatment was considered complete if $500 \mu \mathrm{m}$ size burns on the retina were placed no further than 1 to 2 burn widths apart, beginning two disc diameters temporal to the center of macula and one disc diameter nasal to the optic disc, and extending towards the equator for the 12 clock hours. However, 10 clock hours would be sufficient if remaining VH prevents sufficient treatment of the 12 clock hours. The power was adjusted to produce grayish white reaction and pulse duration was set at 100 milliseconds.

\section{Pars plana vitrectomy (PPV)}

23 gauge PPV was used starting with clearance of anterior vitreous followed by core vitrectomy, peripheral vitrectomy then removal of posterior hyaloid membrane. Vitreous base shaving with 360 scleral depression and endolaser treatment were done for all eyes followed by fluid air exchange, 20\% SF6 was used only if there was active significant bleeding during surgery or if there was intraoperative retinal tears. All eyes received a combination of topical steroid and antibiotic eye drops as postoperative treatment.

Follow up was carried out after one day, one week, one month, two months, three months, six months and nine months following the initial procedure whether IVI or vitrectomy. Patients of group I were scheduled for subsequent IVIs and PRP during their follow up visits. Patients were advised to report immediately if there was any vision deterioration for the possibility of recurrent bleeding.

VH clearance was classified as complete clearance with clear posterior pole, optic disc and peripheral retina enough to deliver complete PRP as described above, partial clearance was considered if there was unclear posterior pole and optic disc or insufficient peripheral retina to deliver complete PRP and persistent or nonclearing $V H$ was considered if the density was the same as time of presentation. Recurrent $V H$ was defined as increase in the density of the hemorrhage after complete or partial clearance in addition to worsening visual acuity in comparison to the previous visit.

\section{Additional treatment for group I}

PPV was done for non-clearing VH or partial clearance that could not enable complete PRP after three months. If recurrent $\mathrm{VH}$ occurred after three IVAs with complete PRP at any time after three months, IVA would be given once more and PPV was indicated if the hemorrhage was not cleared after one month (Fig. 1).

\section{Additional treatment for group II}

Follow up was done for immediate postoperative $\mathrm{VH}$ and IVA was indicated if the hemorrhage was not cleared after one month. If recurrence occurred after the first month, IVA was given with follow up for one month, and if not cleared, pars plana wash of the hemorrhage was indicated (Fig. 2).

The primary outcome measure was change in the mean Log MAR BCVA in both groups after nine months. The secondary outcome measures were duration of clearance of $\mathrm{VH}$, additional required treatment in both groups and report of any complications. 


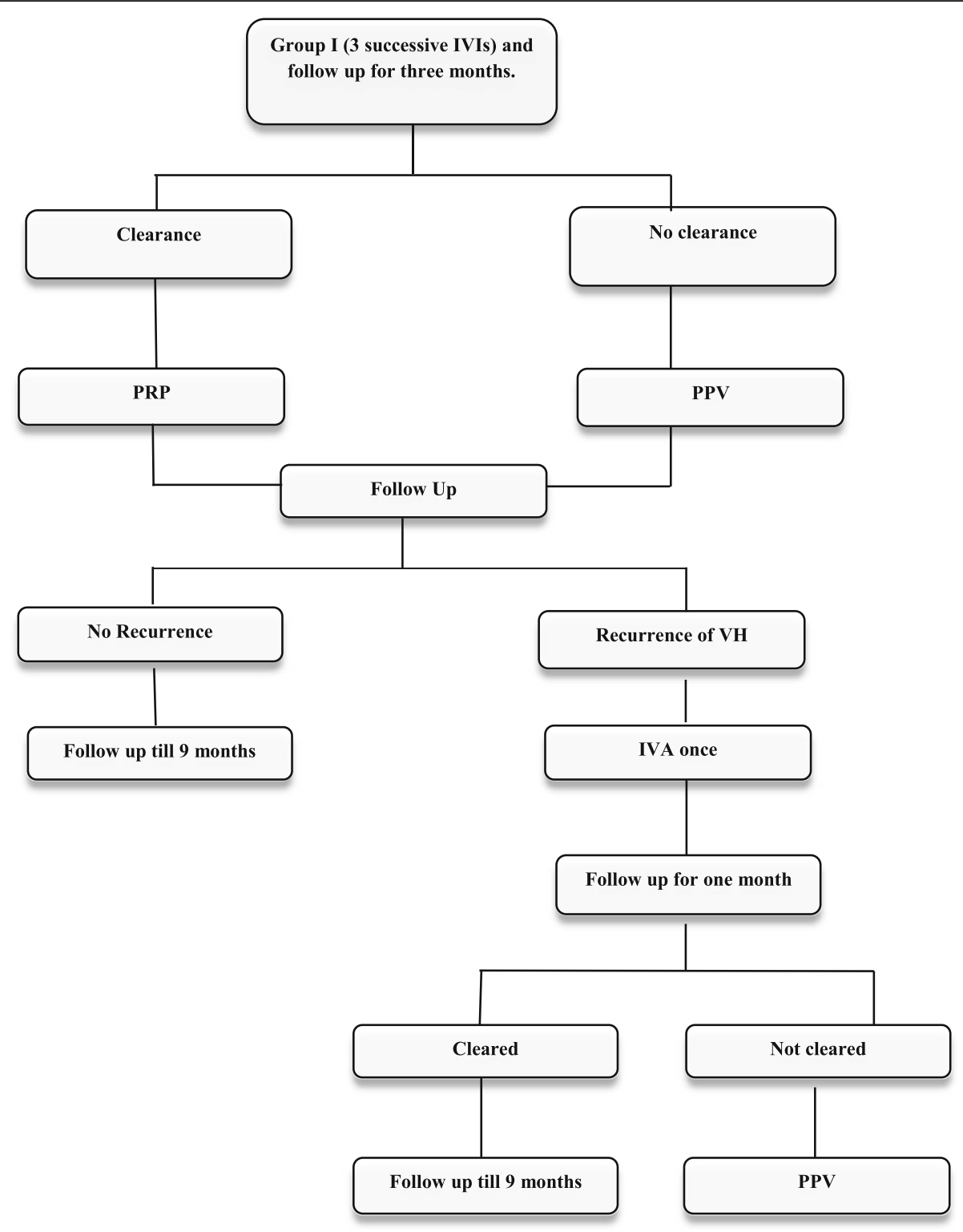

Fig. 1 Flow chart for group I

\section{Data management and statistical analysis}

The collected data was revised and introduced to a PC using Statistical package for Social Science (SPSS, Chicago, inc, USA, version 20). Data was presented and suitable analysis was done according to the type of data obtained for each parameter. Parametric numerical data were expressed as mean and standard deviation (SD). Unpaired $T$ Test was used to assess the statistical significance of the difference between two study groups' means (significant difference was considered if $p \leq 0.05)$. Repeated Measure ANOVA test was used to assess the statistical significance of the same variable measure more than two times among the same group.

\section{Results}

There was no statistically significant difference regarding the initial demographic characteristics between both groups (Table 1).

\section{BCVA changes in both groups}

The difference was not statistically significant regarding the mean initial log MAR BCVA $(1.17 \pm 0.48,1.44 \pm 0.44$ respectively, $P=0.158)$. At one month, the difference was significantly better in group II than group I $(0.65 \pm$ $0.22,0.93 \pm 0.37$ respectively, $p=0.039)$. At two months, the mean log MAR BCVA was also significantly better in group II than group I $(0.49 \pm 0.19,0.71 \pm 0.2$ respectively, $P=0.042)$. The mean BCVA in group I was not 


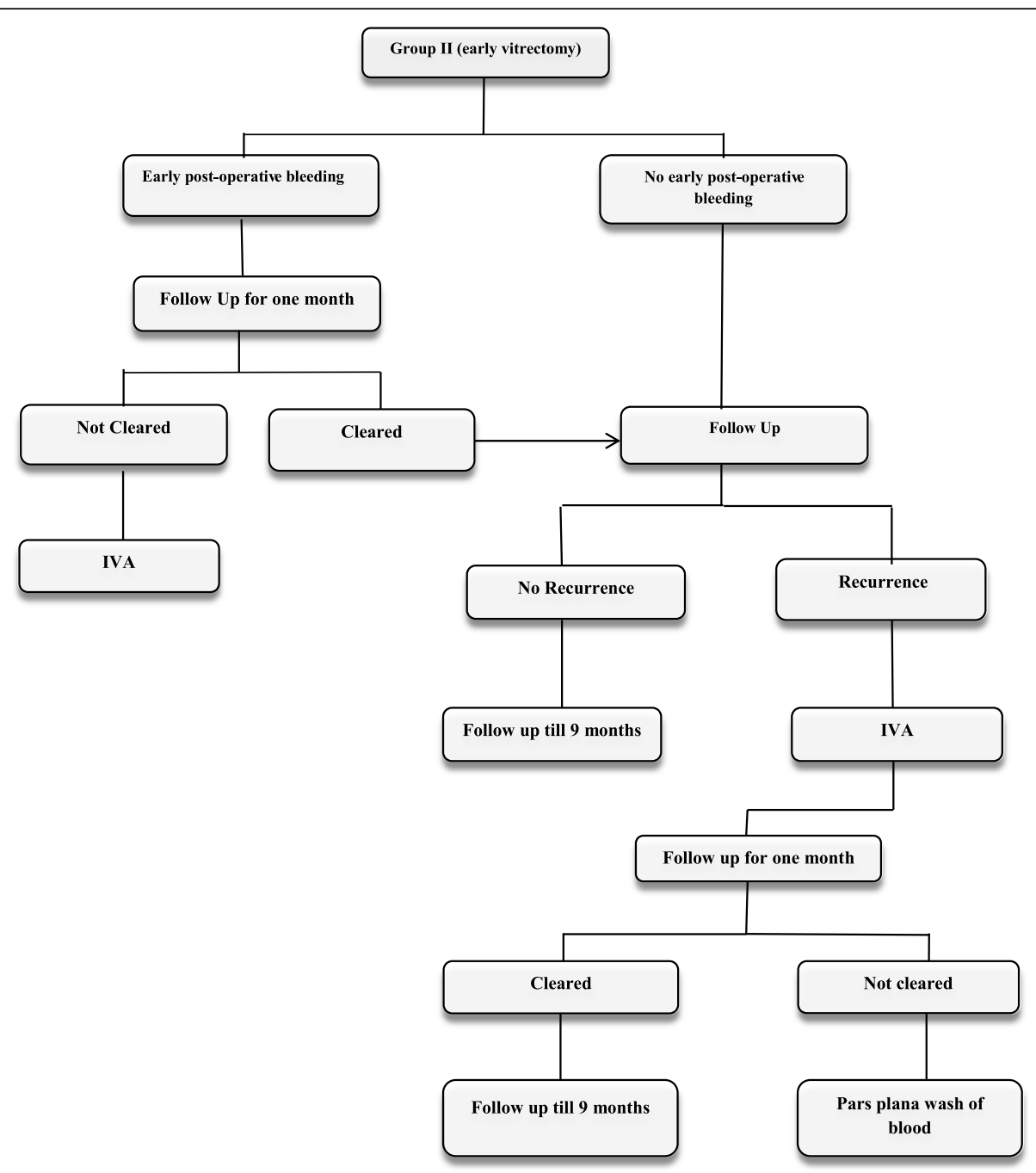

Fig. 2 Flow chart for group II

Table 1 The initial demographic characteristics of both groups

\begin{tabular}{|c|c|c|c|c|c|c|c|}
\hline & & \multicolumn{2}{|c|}{ Group I (17 eyes) } & \multicolumn{2}{|c|}{ Group II (17 eyes) } & \multicolumn{2}{|l|}{$\mathrm{t}$ test } \\
\hline & & Mean/ Nu & $\mathrm{SD} / \%$ & Mean/Nu & $\mathrm{SD} / \%$ & $p$ value & sig. \\
\hline Age (years) & & 56.4 & 8.6 & 58.8 & 7.9 & 0.479 & NS \\
\hline \multirow[t]{2}{*}{ Gender } & Male & 8 & $47.06 \%$ & 9 & $52.94 \%$ & 0.408 & NS \\
\hline & Female & 9 & $52.94 \%$ & 8 & $47.06 \%$ & & \\
\hline \multirow[t]{2}{*}{ DM type } & I & 4 & $23.5 \%$ & 2 & $11.8 \%$ & 0.164 & NS \\
\hline & II & 13 & $76.5 \%$ & 15 & $88.2 \%$ & 0.134 & \\
\hline Duration of DM (years) & & $16.4 \pm$ & 3.8 & $15.8 \pm$ & 3.9 & 0.387 & NS \\
\hline \multirow[t]{3}{*}{ VH Grading } & 1 & 2 & $11.8 \%$ & 2 & $11.8 \%$ & 1 & NS \\
\hline & II & 9 & $52.9 \%$ & 9 & $52.9 \%$ & & \\
\hline & III & 6 & $35.3 \%$ & 6 & $35.3 \%$ & & \\
\hline \multirow[t]{2}{*}{ Lens status } & Phakic & 12 & $70.6 \%$ & 11 & $64.7 \%$ & 0.187 & NS \\
\hline & Pseudophakic & 5 & $29.4 \%$ & 6 & $35.3 \%$ & 0.234 & NS \\
\hline
\end{tabular}


statistically different from group II at 3 months $(0.55 \pm$ $0.2,0.50 \pm 0.17$ respectively, $P=0.508$ ). At six months, the difference between both groups was also insignificant $(0.52 \pm 0.19,0.46 \pm 0.1$ respectively, $p=0.347)$. At nine months, the difference was also insignificant between both groups $(0.51 \pm 0.20,0.48 \pm 0.18$ respectively, $p=0.412)$ (Fig. 3)

The mean final BCVA was statistically better than the initial BCVA in group I $(0.51 \pm 0.20,1.17 \pm 0.48, P<$ 0.001 ) and the mean final BCVA was statistically better than the initial BCVA in group II $(0.48 \pm 0.18,1.44 \pm$ $0.44, P<0.001)$.

There was no significant difference between both groups regarding the number of eyes having final $\log$ MAR BCVA better than $0.5,0.5-0.7$ or worse than 0.7(Fig. 4).

\section{Clearance of VH}

The mean duration of $\mathrm{VH}$ clearance in group I was $7.8 \pm 1.8$ weeks while it was 5 days in group II (SS, $p \leq 0.05)$.

\section{Significant complications}

Epiretinal membrane (ERM) was found in two eyes $(11.8 \%)$ in group I and two eyes (11.8\%) in group II. Cataract occurred in four out of eleven phakic eyes (36.4\%) in group II (uneventful phacoemulsification and intraocular lens implantation was done for these eyes in the time between three and six months). Meanwhile, none of the eyes in group I had cataract. Intraoperative retinal tear occurred in two eyes $(11.8 \%)$ in group II. Significant intraoperative bleeding occurred in one eye (5.9\%) in group II. 20\% SF6 gas was used in three eyes $(17.6 \%)$ in group II. Neither intraocular infection nor glaucoma occurred in any eye in both groups. None of the eyes had retinal detachment.

\section{Additional treatment in both groups for recurrent $\mathrm{VH}$ Group I}

After three months, clearance of VH with complete PRP was achieved in all eyes (100\%). Recurrent $\mathrm{VH}$ occurred in five eyes (29.4\%); the average time for recurrence was 19 weeks. IVA injection was given once after diagnosis of recurrent VH. Two eyes (11.8\%) showed cleared VH within a month after the injection and vitrectomy was done for three eyes (17.6\%) due to non-clearance of $\mathrm{VH}$ after one month from last IVA injection.

\section{Group II}

Early mild postoperative VH was found in 6 eyes (35.3\%) immediately after surgery. All eyes showed complete clearance within the first month, late recurrent VH (after three months) occurred in two eyes (11.8\%), IVA injection was given with complete clearance.

The difference in the recurrence rate between group I $(29.4 \%)$ and group II (11.8\%) was statistically significant $(p<0.05)$.

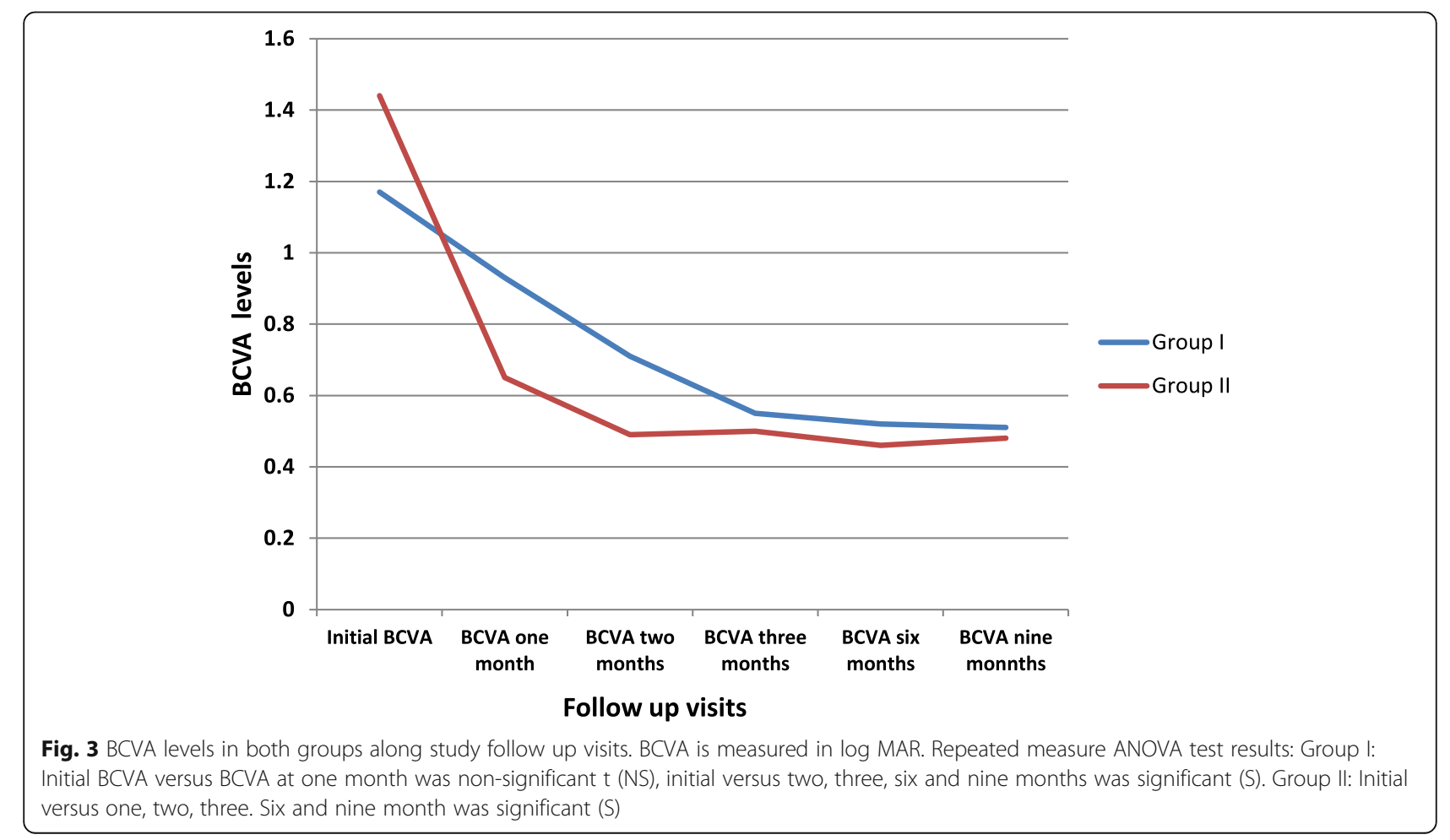




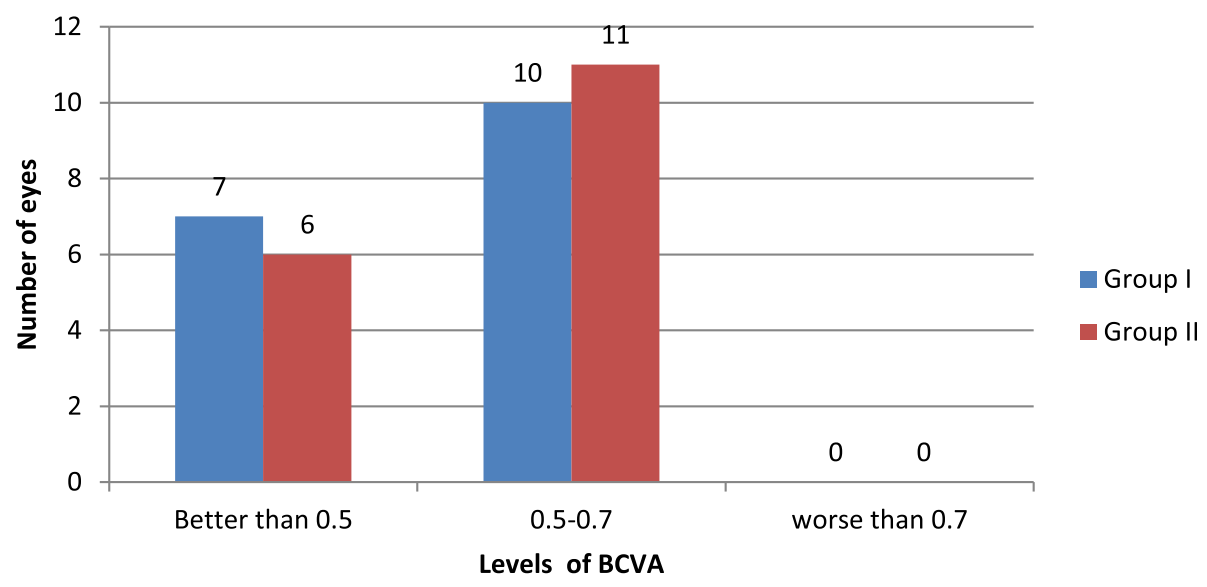

Fig. 4 Number of eyes with different final postoperative visual acuity levels in both groups. BCVA levels measured in Log MAR. Treated eyes were categorized according to final log MAR BCVA into three groups (better than $0.5,0.5-0.7$ and worse than 0.7)

\section{Discussion}

PDR is a common cause of VH. $50 \%$ of PDR patients who do not receive timely treatment develop serious vision loss within 5 years [3]. Sivaprasad et al, [13] studied the effect of repeated aflibercept injection in comparison to standard PRP in cases of PDR without $\mathrm{VH}$ and showed that regression of retinal neovascularization at 2 years was seen in $81 \%$ of aflibercept-treated eyes and $78 \%$ of PRP-treated eyes. $64 \%$ of aflibercepttreated eyes had total regression of neovascularization, compared to $34 \%$ in PRP eyes. Accordingly, the aflibercept group had greater improvement in retinopathy and showed better BCVA than eyes treated with PRP. Gross et al [4] showed that IVR was found to be non-inferior to PRP in PDR without VH at the end of two year study.

The present study showed that there was significant improvement of final BCVA in group I which is comparable to Alagoz et al [14], who used bevacizumab to treat $\mathrm{VH}$, and DRCRN protocol N [5], which showed better visual outcome with IVR in comparison to saline in the short term follow up. Huang et al [15] found that eyes treated with IVB and those not treated showed similar improvements in BCVA in $\mathrm{VH}$ patients who were followed for at least 12 months. In the present study, it was reported that BCVA was improved after IVA in comparison to initial BCVA but it cannot be stated that it would be better than those cases if observed alone as we do not have control group.

Anti-VEGF therapy can be effective for fresh VH; these drugs can cause regression of the neo vessels and prevent recurrent bleeding or new bleeding episode from a new source of neovascularization until spontaneous absorption of the blood occurs, which can permit adequate PRP delivery to the retina with subsequent permanent effect. It reasonable to state that there is correlation between $\mathrm{VH}$ clearance and vision improvement with the use of IVA injection. Eyes with DME were excluded initially and throughout the study if it was possible to diagnose them but it is crucial to note that baseline diagnosis of DME can be missed in eyes with VH. Some patients in group I could have associated DME with $\mathrm{VH}$ at the beginning of the study which was improved after intravitreal injection and this could be an additive factor for vision improvement in these cases beside VH clearance.

The present study showed complete clearance of $\mathrm{VH}$ in $100 \%$ of eyes after three injections, which was comparable to Spaide and Fisher [16], who reported complete clearance of $\mathrm{VH}$ after one month following intravitreal anti-VEGF injections in two cases of PDR. Alagoz et al [14] reported that $86 \%$ of his patients showed clearance of VH and completed PRP. Also, Elias et al [17] reported that $77.7 \%$ had completed PRP at the end of follow up after IVR injection. These results are slightly different from the present study which showed that $100 \%$ of the eyes had complete PRP after three injections. The difference may be explained by the fact that three successive injections were used even with complete clearance of $\mathrm{VH}$ in the present study while Alagoz et al used anti-VEGF only once and Elias et al used repeated IVRs for those eyes with incomplete VH clearance. In addition, there may be a difference in the biological effects of different drugs (aflibercept in the present study and bevacizumab in Alagoz's study and ranibizumab in Elias's study). This may raise a question of whether to inject only once or three successive injections, which is more effective? In case of one injection, it is expected that some patients will clear within a month and others will take longer duration according to many factors as the severity of PDR and density of VH. It is known that after one month there will be no effect of the injected drug so those eyes with non-cleared $\mathrm{VH}$ 
may be subjected to recurrent bleeding exactly as if they were observed for spontaneous clearance. The other option is to inject as needed; meaning, repeated injections are given only if there is incomplete clearance of $\mathrm{VH}$ (as done in Elias et al study) and PRP is done once the hemorrhage is cleared, which may be more convenient for some patients. However, it was preferred in this study to give three successive injections followed by PRP even if $\mathrm{VH}$ had been cleared before the second or third injection in order to have as much as possible constant concentration of aflibercept inside the vitreous cavity for all eyes for three months. This would provide a better chance of $\mathrm{VH}$ clearance and standardize the protocol of treatment for all eyes with thorough explanation to the patients about the importance of regular follow up and repeating the injections even with clearance of $\mathrm{VH}$.

Recurrent VH occurred in five eyes (29.4\%) in group I. DRCR network Protocol N showed that the rate of recurrent $\mathrm{VH}$ was $6 \%$ for ranibezumab treated eyes and $17 \%$ for those eyes who received saline injection however long term recurrence rate cannot be stated in protocol $\mathrm{N}$ [18] as the follow up period was only for 16 weeks.

In the past, there has been considerable debating on the timing of performing vitrectomy in patients with diabetic VH. Findings from the DRVS showed that $25 \%$ of the early vitrectomy group compared to $15 \%$ of the deferral group had a final visual acuity of 20/40 or better after 2 years follow-up. Patients with type I diabetes benefit more from early vitrectomy than patients with type II [8]. Recently, the evolution of small gauge Trans conjunctival vitrectomy is resulting in less operative time, less postoperative inflammation and faster recovery. The development of many surgical instruments with the advancement of equipments and their fluidics encourage most of retinal specialists to do vitrectomy earlier than before. The current study showed that there was significant improvement of BCVA during the follow up visits in comparison to the initial $\mathrm{BCVA}$, which is comparable to other studies. In one series, the mean visual acuity improved from 20/600 preoperatively to $20 / 90$ postoperatively [10]. Another study done by Fassbender et al, [19] showed that early vitrectomy for $\mathrm{VH}$ due to PDR significantly decreases time spent with vision loss.

The present study showed that mean BCVA in group II is better than group I after one and two months $(0.93 \pm 0.37,0.65 \pm 0.22$ after one month $0.71 \pm 0.20 .49 \pm$ 0.19 after two months) which is expected as surgery usually leads to rapid media clearance in comparison to spontaneous absorption of the blood with the aid of anti-VEGF. There was no difference between the two groups after three, six and nine months.
Although both observation and IVI of anti-VEGF can lead to clearance of vitreous hemorrhage after some variable time, early vitrectomy can be done for patients who seek rapid vision regain and are not comfortable with having poor vision even for one eye for many months as in certain occupations such as drivers, even if $\mathrm{VH}$ is being cleared with remaining floater of a blood clot coming intermittently in front of his central vision. Another factor that can make rapid clearance of $\mathrm{VH}$ and subsequently rapid vision regain important is the need to regularly monitor other diabetic retinopathy related problems as macular edema or epiretinal membranes.

To our knowledge, this the first published comparative study between anti-VEGF and early vitrectomy for diabetic VH. Although all eyes in both groups had received $\mathrm{PRP}$, the rate of recurrent $\mathrm{VH}$ is less in group II than group I. This may be explained by the fact that PPV allows removal of any existing traction on any remaining vascular proliferations and allows doing PRP more anterior than slit lamp delivered PRP, which decreases the chance of recurrent bleeding. Early postoperative bleeding occurred in six eyes (35.3\%) in group II, which is comparable to other studies ranging from 20 to 63\% [7] and all the eyes showed mild grade one bleeding. The source of this ooze is usually dispersed blood cells from unremoved parts of peripheral vitreous base, especially in phakic eyes. Sclerotomy site may be another source, and mild hypotony that can occur after suturless PPV can lead to ooze from preexisting retinal neovascularization; however, this mild hemorrhage was cleared spontaneously in all eyes within the first month.

The rate of epiretinal membrane formation was similar between both study groups and none of the eyes needed vitrectomy and membrane peel as they were satisfied with their vision. Cataract formation rate was significantly higher in group II than I but it did not affect the final BCVA as uneventful phacoemulsification and intraocular lens implantation was done. 20\% SF6 gas was used in three eyes (17.6\%). It did not affect the mean BCVA measurement in the first visit after one month as it was completely absorbed by that time.

The current study is limited by the small number of patients in each group and the relatively short period of follow-up.

\section{Conclusions}

Both intravitreal injection of aflibercept and early vitrectomy are effective in treatment of diabetic vitreous hemorrhage with comparable visual outcome. Early vitrectomy can lead to faster vision improvement with less incidence of recurrent hemorrhage, which can be suitable for some patients. Both modalities are equally safe in treatment of diabetic vitreous hemorrhage. 


\section{Abbreviations}

IVA: Intravitreal Aflibercept; IVR: Intravitreal ranibezumab; IVB: Intravitreal bevacizumab; PRP: Panretinal photocoagulation; VH: Vitreous Hemorrhage; BCVA: Best Corrected Visual Acuity; PDR: Proliferative diabetic retinopathy; PPV: Pars plana vitrrectomy; IOP: Intraocular pressure; DRVS: Diabetic retinopathty vitrectomy study; DM: Diabetes Mellitus; IVI: Intravitreal injection; DME: Diabetic macular edema; VEGF: Vascular endothelial growth factor

\section{Acknowledgments}

Not applicable.

\section{Authors' contributions}

AHA conceptualized the research, contributed to preparing the design of the study, contributed to the interventions, contributed to interpretation and analysis of the data and writing the manuscript. AMK contributed to preparing the design of the study. AAM and AMK contributed to the interventions done for participants, the interpretation and analysis of the data and finalization of the manuscript. All authors read and approved the final manuscript.

\section{Funding}

No funding sources.

\section{Availability of data and materials}

Data are available with the author upon reasonable request.

\section{Ethics approval and consent to participate}

The study was approved by the Ethical Committee of Hadi hospital and in accordance with Helsinki declaration. Written informed consent was obtained from all participants.

\section{Consent for publication}

Not applicable.

\section{Competing interests}

The authors declare that they have no competing interests.

\section{Author details}

${ }^{1}$ Ophthalmology Department, Ain Shams University, Cairo, Egypt. ${ }^{2}$ Affiliated as vitreoretinal consultant, Hadi hospital, Jabriya, Kuwait. ${ }^{3}$ Ophthalmology, Mansoura University, Mansoura, Egypt.

\section{Received: 6 November 2019 Accepted: 24 March 2020}

Published online: 06 April 2020

\section{References}

1. Michels RG. Proliferative diabetic retinopathy: pathophysiology of extra retinal complications and principles of vitreous surgery. Retina. 1981;1:1-17.

2. Sinawat S, Rattanapakorn T, Sanguansak T, Yospaiboon Y. Intravitreal bevacizumab for proliferative diabetic retinopathy with new dense vitreous hemorrhage after full panretinal photocoagulation. Eye. 2013;27:1391-6.

3. Simunovic MP, Maberley DA. Anti-vascular endothelial growth factor therapy for proliferative diabetic retinopathy: a systematic review and metaanalysis. Retina. 2015;35:1931-42.

4. Gross JG, Glassman AR, Jampol LM, Inusah S, Aiello LP, Antoszyk AN, Baker CW, Berger BB, Bressler NM, Browning D, Elman MJ, Ferris FL III, Friedman SM, Marcus DM, Melia M, Stockdale CR, Sun JK, Beck RW. Diabetic Retinopathy Clinical Research Network. Panretinal Photocoagulation vs Intravitreous Ranibizumab for Proliferative Diabetic Retinopathy: A Randomized Clinical Trial. JAMA. 2015:314:2137-46.

5. Bshavsar AR, Torres $K$, Glassman RA, et al. Evaluation of results 1 year following short-term use of ranibizumab for vitreous hemorrhage due to proliferative diabetic retinopathy. JAMA Ophthalmol. 2014;132(7):889-90.

6. Parikh RN, Traband A, Kolomeyer AM, VanderBeek BL, Kim BJ, Maguire AM, Brucker AJ. Intravitreal bevacizumab for the treatment of vitreous hemorrhage due to proliferative diabetic retinopathy. Am J Ophthalmol. 2017:176:194-202

7. Khuthaila MK, Hsu J, Chiang A, et al. Postoperative vitreous hemorrhage after diabetic 23-gauge pars plana vitrectomy. Am J Ophthalmol. 2013;15: 757-63
8. Diabetic Retinopathy Vitrectomy Study Research Group. Early vitrectomy for severe vitreous hemorrhage in diabetic retinopathy. Four-year results of a randomized trial: Diabetic Retinopathy Vitrectomy Study Report 5. Arch Ophthalmol. 1990;108:958-64.

9. Virata SR, Kylstra JA. Postoperative complications following vitrectomy for proliferative diabetic retinopathy with sew-on and noncontact wide-angle viewing lenses. Ophthalmic Surg Lasers. 2001;32:193-7.

10. Gupta B, Sivaprasad S, Wong R, et al. Visual and anatomical outcomes following vitrectomy for complications of diabetic retinopathy: the DRIVE UK study. Eye (Lond). 2012;26:510-6.

11. Misra A, Ho-Yen G, Burton RL. 23-gauge sutureless vitrectomy and 20-gauge vitrectomy: a case series comparison. Eye (Lond). 2009;23:1187-91.

12. Nagpal M, Wartikar S, Nagpal K. Comparison of clinical outcomes and wound dynamics of sclerotomy ports of 20,25, and 23 gauge vitrectomy. Retina. 2009:29:225-31.

13. Sivaprasad S, Hykin P, Prevost AT, Vasconcelos J, Riddell A, Ramu J, Murphy C, Kelly J, Edwards RT, Yeo ST, Bainbridge J, Hopkins D. Intravitreal aflibercept compared with panretinal photocoagulation for proliferative diabetic retinopathy: the CLARITY non-inferiority RCT. NIHR Journals Library: Southampton (UK); 2018 Oct.

14. Alagöz C, Yıldırım Y, Kocamaz M, Baz Ö, Çiçek U, Çelik B. Halil Ibrahim Demirkale, Ahmet Taylan Yazıcı, and Muhittin Taşkapılı. The efficacy of Intravitreal Bevacizumab in vitreous hemorrhage of diabetic subjects. Turk J Ophthalmol. 2016:46:221-5.

15. Huang $\mathrm{YH}$, Yeh PT, Chen MS, et al. Intravitreal bevacizumab and panretinal photocoagulation for proliferative diabetic retinopathy associated with vitreous hemorrhage. Retina. 2009;29:1134-40.

16. Spaide RF, Fisher YL. Intravitreal bevacizumab (Avastin) treatment of proliferative diabetic retinopathy complicated by vitreous hemorrhage. Retina. 2006;26:275-8.

17. Chelala EL, Nehme J, Rami H, Aoun R, Dirani A, Fadlallah A, Jalkh A. Efficacy of Intravitreal Ranibizumab injections in the treatment of vitreous hemorrhage related to proliferative diabetic retinopathy. J Retinal Vitreous Dis. 2018;38:1127-33.

18. Randomized Clinical Trial Evaluating Intravitreal Ranibizumab or Saline for Vitreous Hemorrhage from Proliferative Diabetic Retinopathy, Bhavsar AR, Torres K, Beck RW, Bressler NM, Ferris FL III, Friedman SM, Glassman AR, Maturi RK, Melia M, Singer MA, Stockdale CR. Diabetic Retinopathy Clinical Research Network. JAMA Ophthalmol. 2013;131:283-93.

19. Fassbender JM, Ozkok A, Canter H, Schaal S. A comparison of early and delayed vitrectomy for management of vitreous hemorrhage due to proliferative diabetic retinopathy. Investigative Ophthalmology \& Visual Science an ARVO Journal. 2015;56:5117-42.

\section{Publisher's Note}

Springer Nature remains neutral with regard to jurisdictional claims in published maps and institutional affiliations.

Ready to submit your research? Choose BMC and benefit from:

- fast, convenient online submission

- thorough peer review by experienced researchers in your field

- rapid publication on acceptance

- support for research data, including large and complex data types

- gold Open Access which fosters wider collaboration and increased citations

- maximum visibility for your research: over $100 \mathrm{M}$ website views per year

At $\mathrm{BMC}$, research is always in progress.

Learn more biomedcentral.com/submission 